\title{
RINGS WITH THE CONTRACTION PROPERTY
}

\section{WILLIAM J. WICKLESS ${ }^{1}$}

AbSTRACT. A ring $R$ (not necessarily commutative or with unit) has the contraction property iff every ideal of every subring of $R$ is a contracted ideal.

It is shown that $R$ is a primitive ring with the contraction property iff $R$ is an absolutely algebraic field. This result, together with the fact that the Jacobson Radical of a ring with the contraction property is nil, shows that a nil semisimple ring with the contraction property is a subdirect sum of absolutely algebraic fields (and is therefore commutative).

It is shown that if $R$ is a torsion free nil ring with the contraction property then $R^{2}=(0)$. It follows that any torsion free ring with the contraction property is the extension of a zero ring and a subdirect sum of absolutely algebraic fields. Also, if $R$ is a nil ring with the contraction property then $R^{2}$ is torsion as an additive group.

\section{Rings with the contraction property.}

Definition 1.1. A ring $R$ (not necessarily commutative or with unit) has the contraction property iff every ideal of every subring of $R$ is a contracted ideal; that is, if $S$ is a subring of $R$ and $I$ an ideal of $S$, we have $I=T \cap S$ for some ideal $T$ of $R$ [6, pp. 218-221]).

Hereafter, we call $R$ a $c$-ring iff it satisfies the condition of Definition 1.1. The ring of integers is a simple example of a $c$-ring. Another example is any absolutely algebraic field $F[3$, p. 147], for if $F$ is any absolutely algebraic field and $S$ is any subring of $F$, it is easy to check that $S$ is actually a subfield of $F$. Thus, the only ideals of $S$ are $(0)$ and $S$ which are contracted ideals.

THEOREM 1.1. The class of all c-rings is closed under homomorphisms, direct sums, if each summand is a ring with unit, and is hereditary for subrings.

Proof. (1) Let $A$ be a $c$-ring and $B$ a homomorphic image of $A$ via a homomorphism $\varphi$. Let $S$ be a subring of $B, I$ an ideal of $S . \varphi^{-1}(I)$ is an ideal in $\varphi^{-1}(S)$, a subring of $A$, thus $\varphi^{-1}(I)=T \cap \varphi^{-1}(S)$, with

Received by the editors February 16, 1970 and, in revised form, April 17, 1970.

AMS 1969 subject classifications. Primary 1620, 1932, 1642.

Key words and phrases. Contracted ideal, nil ring, absolutely algebraic field. $\$ 13164$.

1 The preparation of this paper was sponsored in part by the NSF Grant GP 
$T$ an ideal of $A$. Let $T^{\prime}=(T+\operatorname{Ker} \varphi)$. It is easy to check that $I$ $=\varphi\left(T^{\prime}\right) \cap S$.

(2) Let $\left\{A_{\alpha} \mid \alpha \in V\right\}$ be a set of $c$-rings with unit and $A$ $=\sum_{\alpha \in V} \oplus A_{\alpha}$. Let $S$ be a subring of $A, I$ an ideal in $S$. Let $\pi_{\alpha}$ be the projection of $A$ onto $\bar{A}_{\alpha}$, the subring of $A$ naturally isomorphic to the ring $A_{\alpha}$. For each $\alpha \pi_{\alpha}(I)$ is an ideal in $\pi_{\alpha}(S)$, a subring of the $c$-ring $\bar{A}_{\alpha}$. Thus, for all $\alpha$ we have $\pi_{\alpha}(I)=\pi_{\alpha}(S) \cap \bar{I}_{\alpha}$, where $\bar{I}_{\alpha}$ is an ideal in $\bar{A}_{\alpha}$. Let $\bar{I}=\sum_{\alpha \in V} \oplus \bar{I}_{\alpha}$. It is now easy to check, as each $A_{\alpha}$ is a ring with unit, that $\bar{I}$ is an ideal in $A$ with $\bar{I} \cap S=I$.

(3) Let $B$ be a subring of a $c$-ring $A, S$ be a subring of $B, I$ be an ideal in $S$. As $S$ is also a subring of $A$, we have $I=\bar{I} \cap S$ for $\bar{I}$ an ideal of $A$. But then, clearly, $I=(\bar{I} \cap B) \cap S$, so $B$ is also a $c$-ring.

2. c-rings without nil ideals. We next consider $c$-rings without nil ideals. First we classify all primitive $c$-rings.

TheOREM 2.1. $R$ is a primitive c-ring iff $R$ is an absolutely algebraic field.

Proof. We have already noted that any absolutely algebraic field is a $c$-ring.

Now let $R$ be any primitive $c$-ring. Let $M$ be an irreducible $R$ module with centralizer $\Gamma$. We first note that $\operatorname{dim}(M: \Gamma)=1$, otherwise we could find a subring $S \subset R$ which could be mapped homomorphically onto $(\Gamma)_{2 \times 2}$-the ring of all $2 \times 2$ matrices with entries from $\Gamma$, $[4$, p. 33, Theorem 3]. But this, together with Theorem 1.1, would imply that $(\Gamma)_{2 \times 2}$ is a $c$-ring-a contradiction, since

$$
\left\{\left(\begin{array}{ll}
0 & 0 \\
a & 0
\end{array}\right)\right\} \text { is an ideal in the subring }\left\{\left(\begin{array}{ll}
b & 0 \\
a & c
\end{array}\right)\right\}
$$

which is not a contracted ideal. As $\operatorname{dim}(M: \Gamma)=1$ we have $R=\Gamma, \Gamma$ a division ring.

We next note that $\Gamma$ has characteristic $p \neq 0$, since otherwise $Z$, the ring of integers, would be a subring of $\Gamma$. As $\Gamma$ is simple, no proper ideal of $Z$ could be contracted-a contradiction.

Let $Z_{p}$ denote the prime field of $\Gamma$. For all $x \in \Gamma, x$ must be algebraic over $Z_{p}$, since otherwise the polynomial ring $Z_{p}[x]$ would be a subring of $\Gamma$ in which no proper ideal was contracted. Finally, as $Z_{p}[x]$ is a finite field, for all $x \in \Gamma$ we have $x^{p^{k}}=x$ where $k$ $=\operatorname{dim}\left(Z_{p}[x]: Z_{p}\right)$. But this means that $\Gamma$ must be commutative $[2, \mathrm{p}$. 72 ]. Thus, $R=\Gamma$ is an absolutely algebraic field.

In [5, Theorem 6], we proved that the Jacobson Radical of any $c$-ring is nil. This, together with 2.1 , gives the following result. 
THEOREM 2.2. Let $R$ be a c-ring without nil ideals. Then $R$ is a subdirect sum of absolutely algebraic fields (and is therefore commutative).

3. Nil $c$-rings. First we consider nilpotent $c$-rings.

TheOREM 3.1. Let $R$ be a torsion free nilpotent c-ring. Then $R^{3}=(0)$.

Proof. Let $R$ be a nilpotent $c$-ring of index $k \geqq 4$. Choose $x_{1}$, $x_{2}, \cdots, x_{k-1}$ in $R$ such that $x_{1} x_{2} \cdots x_{k-1} \neq 0$. The set of all integral multiples of $x_{1} \cdots x_{k-2}$ is a subgroup of $R^{2}$ as $k \geqq 4$. As $R^{k}=(0)$ this subgroup is moreover an ideal in $R^{2}$. As $R$ is a $c$-ring this subgroup must actually be an ideal in $R$. Thus, we have $x_{1} \cdots x_{k-2} \cdot x_{k-1}$ $=m x_{1} \cdots x_{k-2}$ for some integer $m$. This yields $m x_{1} \cdots x_{k-1}=0$, a contradiction since $R$ is torsion free.

TheOREM 3.2. Let $R$ be a torsion free nil c-ring. Then $R^{3}=(0)$.

Proof. As $R$ is a torsion free nil $c$-ring, for all $x \in R,\langle x\rangle$, the subring generated by $x$, is a torsion free nilpotent $c$-ring. Thus $x \in R$ implies $x^{3}=0$. We now can conclude that $R$ is locally nilpotent [ 1 , p. 130]. Let $u, v, w$ be any elements of $R$. $S$, the subring generated by $u, v, w$ is a torsion free nilpotent $c$-ring. Applying 3.1 we have $S^{3}$ $=(0)$. Thus, $u v w=0$ and we have $R^{3}=(0)$.

THeOREM 3.3. Let $R$ be a torsion free nil c-ring. Then $R^{2}=(0)$.

Proof. First we note that $x^{2}=0$ for every $x \in R$. To see this, let $\langle x\rangle$ be the subring generated by $x$ and $m$ an integer with $m>1$. I $=\left\{t m x+l m^{2} x^{2} \mid t, l \in Z\right\}$ is an ideal of $m\langle x\rangle$. As $\langle x\rangle$ is a $c$-ring and $m\langle x\rangle$ is an ideal in $\langle x\rangle$ we see that $I$ must be an ideal in $\langle x\rangle$. Thus $m x^{2}=t m x+l m^{2} x^{2}$ for some $t, l \in Z$. Multiplying by $x$ we have $t m x^{2}=0$. If $t \neq 0$, as $R$ is torsion free, we have $x^{2}=0$. If $t=0$ then $\left(l m^{2}-m\right) x^{2}=0$ and $l m^{2}-m \neq 0$ so $x^{2}=0$.

Now let $u, v$ be arbitrary elements of $R$. We have $u v+v u=(u+v)^{2}$ $-u^{2}-v^{2}=0$, so $u v=-u v$. Let $S$ be the subring of $R$ generated by $u$ and $v$. Now $L=\{t u+s u v \mid t, s \in Z\}$ is an ideal in $S$ since $S^{3}=(0)$ and $u v=-v u$. Also, $K=\{u \mid t \in Z\}$ is an ideal in $L$ since $K L=L K=(0)$. As $S$ is a $c$-ring, we have $K$ is an ideal in $S$. Thus $u v=t u$ for some $t \in Z$. If $t=0$ then $u v=0$; if $t \neq 0$ multiplying by $v$ yields $t u v=0$ and again $u v=0$ as $R$ is torsion free. As $u, v$ were arbitrary we have $R^{2}=(0)$.

CoROLlaRy. Let $R$ be a torsion free c-ring. Then $R$ is the extension of a zero ring and a subdirect sum of absolutely algebraic fields.

Corollary. Let $R$ be a nil c-ring. Then $R^{2}$ is torsion as an additive group. 


\section{REFERENCES}

1. N. J. Divinsky, Rings and radicals, Math. Expositions, no. 14, Univ. of Toronto Press, Toronto, 1965. MR 33 \#5654.

2. I. N. Herstein, Noncommutative rings, Carus Math. Monographs, no. 15, Math. Assoc. of America, distributed by Wiley, New York, 1968. MR 37 \#2790.

3. N. Jacobson, Lectures in abstract algebra. Vol. 3: Theory of fields and Galois theory, Van Nostrand, Princeton, N. J., 1964. MR 30 \#3087.

4. - Structure of rings, 2nd ed., Amer. Math. Soc. Colloq. Publ., vol. 37, Amer. Math. Soc., Providence, R. I., 1964. MR 36 \#5158.

5. W. J. Wickless, $A$ characterization of the nil radical of a ring, Pacific J. Math. (to appear).

6. O. Zariski and P. Samuel, Commutative algebra. Vol. 1, University Series in Higher Math., Van Nostrand, Princeton, N. J., 1958. MR 19, 833.

University of California, Los Angeles, California 90024 